\title{
Free Molecular Flows Between Two Plates Equipped with Pumps
}

\author{
Chunpei Cai* \\ ZONA Technology, Inc., Scottsdale, Arizona 85258 \\ Iain D. Boyd $\ddagger$ \\ University of Michigan, Ann Arbor, Michigan 48109 \\ and \\ Quanhua Sun $¥$ \\ ESI US R\&D, Inc., a Member of ESI Group, Huntsville, Alabama 35806 \\ DOI: $10.2514 / 1.25891$
}

\begin{abstract}
This study describes a theoretical analysis of free-molecular flows between two plates, both equipped with vacuum pumps, with an aim to investigate the facility effects of large chambers equipped with distributed cyrogenic vacuum pumps. Three free-molecular models are proposed to study the unsteady flow behavior, and to calculate the average number density and average velocity of the rarefied background gas molecules between the two plates. The first model is based on mass conservation and predicts that the unsteady evolution is a multiple time scale process. The second model is a classical steady kinetic model, whereas the third model is the most advanced kinetic model that includes chamber wall effects. In the last two models, a detailed balance of flux along different flow directions and reflection relations lead to exact solutions. All three models include the chamber facility effects, especially pump size and pump sticking coefficient. Simulations with the direct simulation Monte Carlo method are performed and the results are compared with the analytical results. The analytical results from these models can be used to estimate the average background pressure and velocity in large vacuum chambers equipped with multiple pumps for experiments and simulations.
\end{abstract}

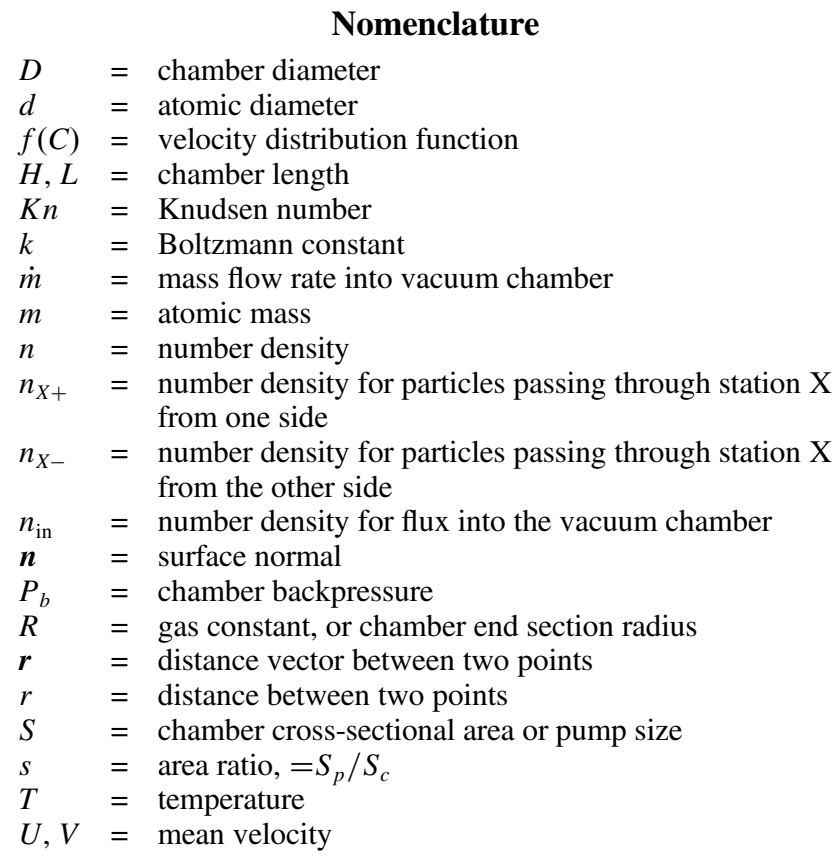

Presented as Paper 3802 at the 9th AIAA/ASME Joint Thermophysics and Heat Transfer Conference, San Francisco, California; received 14 June 2006; revision received 29 August 2006; accepted for publication 29 August 2006. Copyright $@ 2006$ by the American Institute of Aeronautics and Astronautics, Inc. All rights reserved. Copies of this paper may be made for personal or internal use, on condition that the copier pay the $\$ 10.00$ per-copy fee to the Copyright Clearance Center, Inc., 222 Rosewood Drive, Danvers, MA 01923; include the code $\$ 10.00$ in correspondence with the CCC.

${ }^{*}$ CFD Specialist, 8489 E. Ironwood Square Drive; previously graduate student research assistant, Department of Aerospace Engineering, University of Michigan, Ann Arbor, Michigan, AIAA Member.

${ }^{\dagger}$ Professor, Department of Aerospace Engineering, 1320 Beal Avenue, AIAA Associate Fellow.

${ }^{\ddagger}$ Research Scientist, 6767 Old Madison Pike, AIAA Senior Member.

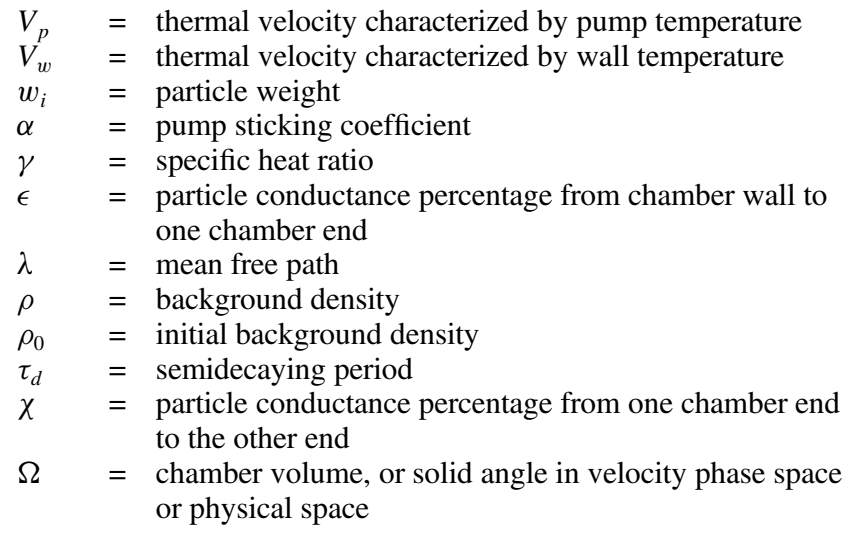

subscript

$\begin{array}{ll}b & =\text { background } \\ c & =\text { chamber } \\ \text { in } & =\text { inflow } \\ w & =\text { wall } \\ 1,2 & =\text { pump } 1 \text { or } 2 \\ A, B & =\text { chamber end } \mathrm{A}, \mathrm{B}\end{array}$

\section{Introduction}

$\mathbf{V}$ ACUUM chambers have wide applications for a variety of purposes such as materials processing and testing of spacecraft electric propulsion systems. The main goal of vacuum chambers is to provide and maintain a low pressure environment. For example, in experiments of testing a cluster of high-power electric propulsion (EP) devices, such as plasma thrusters, vacuum chambers are usually equipped with cryopumps [1-3], and the backpressure in the vacuum chamber is maintained at about $10^{-3}-10^{-4} \mathrm{~Pa}$ with the thrusters in operation. At such a low pressure, the background flow inside the vacuum chamber is usually free-molecular. 
One fundamental concern about the vacuum chamber is to understand the effects of the chamber characteristics on the chamber performance, such as the average background pressure and average velocity in the chamber. These facility characteristics include the pump size, the pump sticking coefficient, the sidewall length, the chamber wall temperature which is usually $300 \mathrm{~K}$, and the cyrogenic pump temperature which is usually maintained at 10-20 K. These facility characteristics can have significant impacts on the vacuum chamber background flow and it is important to properly quantify these facility effects. For example, in EP experiments with large vacuum chambers generally equipped with multiple cyrogenic pumps, the real cyrogenic pump sticking coefficient can be much lower than its nominal value, because there will generally be a propellant frost built up on the cryogenic pumps [4]. For this situation, to accurately estimate the actual sticking coefficient, it is crucial to accurately predict the background pressure and background velocity in the chamber. More generally speaking, a study of facility effects can help to answer the following questions:

1) For a given set of thruster operating conditions and given facility characteristics, what values can be expected for the average background pressure and average background velocity in a chamber? It is crucial to have a clear understanding of this question because the background pressure and background velocity are the two major properties indicating the performance disparity for EP test devices between experimental conditions and real space operation conditions. In space, the ambient pressure and velocity is zero, whereas in a large vacuum chamber, there is always a finite background pressure, and a background average velocity towards the pumps.

2) What are the exact effects of the facility on the background gas?

3) What is the optimal condition that a facility can achieve by adjusting facility properties? Answering these three questions can provide guidelines to improve existing chambers or to design new chambers.

A study of the background flow can have significant influence on numerical simulations as well. For example, in particle simulations of plasma plume flows in large vacuum chambers, usually a large full-scale 3-D simulation is replaced by a simple two-dimensional or axis-symmetric simulation. In such a simplified simulation, traditionally the background flow is replaced with static particles whose velocities are assigned from a zero-centered Maxwellian distribution. However, as pointed out by our previous study [5-7], in chambers equipped with one-sided pumps at one chamber end, the real background flow usually does not follow a zero-centered Maxwellian distribution, and under extreme situations, the average background velocity of the background particles can reach over $100 \mathrm{~m} / \mathrm{s}$. Another interesting result from our previous study is the semidecaying period formula for the unsteady background flow. For large vacuum chambers, such as the large vacuum test facility at the University of Michigan, several seconds are required for the background flow to reach a steady-state condition, hence it is a challenge to perform three-dimensional particle simulations of plume flows in the chamber with a background flow [6,7]. The results of this study will be helpful for numerical simulations of plume flows in vacuum chambers.

Recently, we performed several studies on the facility of large chambers [5-7]. These studies investigated the background flow using simple models of free-molecular flows between two plates equipped with one-sided or two-sided cyrogenic pumps on or near one chamber end. The background flow is separated from the main plume flow inside the chamber. Through the top plate, a net gas flow rate is assumed to enter the chamber, which represents the reflected plume flow at one chamber end, and the thermal speed of the gas flow is characterized by the wall temperature. On the other plate equipped with pumps which are maintained at a low pump temperature, when particles hit the pump surface, they will either stick to the pump and thus be removed with a probability of $\alpha$, or reflect into the chamber with a probability of $1-\alpha$ having a thermal speed characterized by the pump temperature. Analyzing the fluxes along two directions and the number density relations at different locations leads to complete

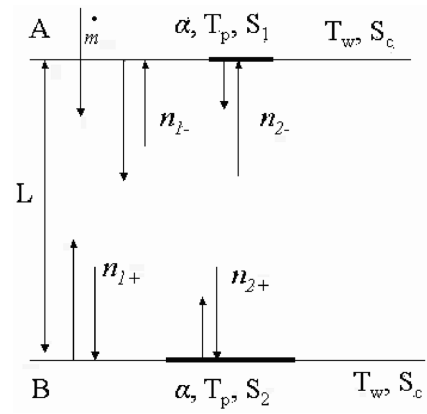

Fig. 1 Illustration of the flow problem for models 1 and 2.

solutions of the background flow, including expressions for the velocity distribution function and the number density.

This article reports our recent progress in a continuing investigation of facility effects for large vacuum chambers equipped with distributed cyrogenic pumps. The problem studied is the freemolecular flow between two plates both equipped with pumps, which is a fair approximation for large vacuum chambers with distributed pumps. For example, the $12 \mathrm{~V}$ chamber at Arnold Engineering Development Center [8] is a large chamber with distributed pumps: there are large cryogenic pump plates located at the bottom of the chamber working as the primary pumps and smaller pumps working as the secondary pumps on the top of the chamber. The problem is illustrated by Fig. 1: through the top plate of area $S_{c}$, there is a diffusion flow characterized by the wall temperature and a mass flow rate into the space between the two plates; both plates are equipped with pumps; the pumps are characterized by individual pump areas $S_{1}$ and $S_{2}$, the same pump temperature, and the same pump sticking coefficient. Particles move between the two plates freely. When the particles strike a wall, they reflect with a thermal velocity characterized by the wall temperature. If particles strike a pump surface, then by a probability of $\alpha$ the particles stick to the pump, or by a probability of $1-\alpha$ they reflect back towards the other plate with a thermal speed characterized by the pump temperature. Note for the reflections on pumps and walls, complete accommodations with the wall or pump surface are assumed. Sidewall effects are included in this study as well. To simplify the study and without lose generality, we assume the top plate is small and the size is set to a fixed value $S_{1} / S_{c}=0.2$. For this study, we still use the assumption that the plume flow does not directly hit the pump.

In real applications such as chambers for EP device experiments, the chamber is usually quite long, $L / R>1$. The chamber length is one of the facility characteristics that can have a significant effect on the background flow, and we classify the chamber to be a short chamber or a long chamber based on the ratio of chamber length to chamber radius for a cylindrical chamber [5,7]. The long chamber wall effects will be discussed with two approaches illustrated in our previous study [5-7]. In the first approach, sidewall length effect is summarized with a specific parameter representing the percentage of particles traveling from one chamber end to the other chamber end without any collisions with the chamber side walls. The other treatment is to simply set the ratio between the wall and pump plate temperatures, $T_{w} / T_{p}$, to unity. This second approach is based on the fact that in long wall situations, almost all particles reflected from the pumps on one chamber end will collide with the chamber wall before they arrive at the other chamber end; hence when they arrive at the other chamber end, their thermal velocities are characterized by the wall temperature instead of the pump temperature. Details of these two approaches can be found in our previous study [ㅁ-] ], and both of them will be applied in this study as well.

The following sections present the results of several analytical models and numerical simulations to validate the analytical solutions. It is worthy to mention that this is a well-defined classical free-molecular flow problem with realistic application background. Even though it will be shown that the solutions to this problem are rather complex, these solutions are more general than those for the problem of one-sided pumps which we investigated previously [ $[5,7]$. The analytical results from this study are applicable to estimate the 
average background number density and velocities, which is significant for the evaluation of vacuum chamber performance and particle simulations of plume flows in a vacuum chamber. To our knowledge, there are similar analytical results in the literature.

\section{Model 1: From Mass Conservation Relation}

Similar to our previous work [5,7], this study assumes a constant density distribution between the two plates equipped with one-sided pumps. Using the mass conservation law for gas in the chamber, the following relation must hold:

$$
\frac{\mathrm{d} \rho}{\mathrm{d} t}=\frac{\mathrm{d}\left(\int \rho \mathrm{d} v\right)}{\Omega \mathrm{d} t}=\frac{1}{\Omega}\left(\dot{m}-\frac{1}{4} \rho \alpha S_{1} V_{1}-\frac{1}{4} \rho \alpha S_{2} V_{2}\right)
$$

where $V_{1}$ and $V_{2}$ are the average thermal velocities towards pumps 1 and 2 with pump surface areas $S_{1}$ and $S_{2}$, respectively. This ordinary differential equation is solved using the following two boundary conditions:

1) At time $t=0$, gas begins to effuse into the chamber through the top plate, the pumps begin to operate, and the average density between the gap is $\rho_{0}$, hence $\rho(t=0)=\rho_{0}$.

2) When the time is sufficiently large, a steady flow is established in the chamber: $\mathrm{d} \rho(t \rightarrow \infty) / \mathrm{d} t=0$.

The solution for this equation is expressed as Eq. (2), which consists of one unsteady term and one steady term:

$$
\begin{aligned}
& \rho(t)=\left[\rho_{0}-\frac{4 \dot{m}}{\alpha\left(S_{1} V_{1}+S_{2} V_{2}\right)}\right] \exp \left[-\frac{\alpha\left(S_{1} V_{1}+S_{2} V_{2}\right)}{4 \Omega} t\right] \\
& +\frac{4 \dot{m}}{\alpha\left(S_{1} V_{1}+S_{2} V_{2}\right)}
\end{aligned}
$$

Pressure in a vacuum chamber is generally measured with an ionization gauge. The pressure corresponding to such experimental measurements [1] is:

$$
\begin{aligned}
& P_{b}(t)=\rho(t) R T_{w}=\left[\rho_{0} R T_{w}\right. \\
& \left.-\frac{4 \dot{m}}{\alpha\left(S_{1} V_{1}+S_{2} V_{2}\right)} R T_{w}\right] \exp \left[-\frac{\alpha\left(S_{1} V_{1}+S_{2} V_{2}\right)}{4 \Omega} t\right] \\
& \quad+\frac{4 \dot{m}}{\alpha\left(S_{1} V_{1}+S_{2} V_{2}\right)} R T_{w}
\end{aligned}
$$

At steady state, for fixed chamber properties, the pressure is proportional to the mass flow rate:

$$
P_{b} S_{c} /\left(\dot{m} \sqrt{2 R T_{w}}\right)=2 \sqrt{2 R T_{w}} S_{c} /\left[\alpha\left(S_{1} V_{1}+S_{2} V_{2}\right)\right]
$$

If the backpressure and the mass flow rate are known, for example, through experimental measurements, then the pump sticking coefficient can be calculated using the following relation:

$$
\alpha=4 \dot{m} R T_{w} /\left[P_{b}\left(S_{1} V_{1}+S_{2} V_{2}\right)\right]
$$

Unfortunately, by including the top pump, this simple model cannot provide an expression for the average velocity between the two plates, though it can be reasoned that the net flow direction is towards the bottom plate as long as the bottom pump has a nonzero size and a nonzero sticking coefficient.

\section{A. Determining the Thermal Speeds $V_{1}$ and $V_{2}$}

\section{Short Chamber Situation}

For a short chamber case, the expressions for $V_{1}$ and $V_{2}$ are complex. With the existence of the small pump on the top plate, along both directions, a fraction of particles have thermal velocities characterized by the wall temperature $V_{w}=\sqrt{8 R T_{w} / \pi}$, and a fraction have thermal velocities characterized by the pump temperature $V_{p}=\sqrt{8 R T_{p} / \pi}$.

A reasonable expression for $V_{1}$ can be constructed by careful consideration of the following facts:
1) When $S_{2}=0$, all particles moving towards pump 1 have a thermal velocity $V_{1}=V_{w}$.

2) When $S_{2}=S_{c}$ and $\alpha=0$, all particles moving towards pump 1 have a thermal velocity $V_{1}=V_{p}$.

3) When $S_{2}=S_{c}$ and $\alpha=1$, all particles hitting pump 2 are absorbed and no particles move towards pump 1. Hence the average velocity is $V_{1}=0$.

With consideration of the above three facts, the simplest linear construction for $V_{1}$ is

$$
\begin{aligned}
V_{1} & =\frac{1}{1-\alpha S_{2} / S_{c}}\left[\left(1-S_{2} / S_{c}\right) \sqrt{8 k T_{w} /(\pi m)}+(1\right. \\
& \left.-\alpha)\left(S_{2} / S_{c}\right) \sqrt{8 k T_{p} /(\pi m)}\right]
\end{aligned}
$$

Constructing $V_{2}$ can follow a similar approach. There are three groups of particles moving towards pump 2: one group consists of particles that entered directly through the top plate; one group is resulted from reflections on the top plate wall; and the last group is due to reflections on the top pump.

1) When $S_{1}=0$, there is no pump on the top plate, and all particles moving towards pump 2 have a thermal velocity $V_{2}=V_{w}$.

2) When $S_{1}=S_{c}$ and $\alpha=0$, all particles reflected from the top plate towards pump 2 have a thermal velocity $V_{p}$, while there is another group of particles with a mass flow rate that moves with a thermal speed $V_{w}$.

3) When $S_{1}=S_{c}$ and $\alpha=1$, all particles hitting the top pumps are absorbed and no particles are reflected towards the bottom pump. Hence, the thermal velocity for this situation is $V_{2}=V_{w}$.

With a consideration of the above three effects, a proper construction for $V_{2}$ is

$$
\begin{aligned}
V_{2} & =\left[1-A(1-\alpha) S_{1} / S_{c}\right] \sqrt{8 k T_{w} /(\pi m)} \\
& +A(1-\alpha)\left(S_{1} / S_{c}\right) \sqrt{8 k T_{p} /(\pi m)}
\end{aligned}
$$

where $A$ is a parameter to be determined.

\section{Long Chamber Situation}

Under the long chamber situation, the expressions for $V_{1}$ and $V_{2}$ are greatly simplified as $V_{1}=V_{2}=\sqrt{8 R T_{w} / \pi}$.

Obviously, though the $V_{1}$ and $V_{2}$ expressions in the short chamber situation are more accurate, they are more complex for practical purposes, especially with the need to determine the parameter $A$ for $V_{2}$. Hence, for simplicity, in this study, we adopt the velocity expressions for long chamber wall situations. This treatment greatly simplifies the final results with a decreased accuracy. However, model 1 is a relatively crude model from which only approximate trends are expected.

\section{B. Discussion}

This crude model, especially Eq. (3), relates several facility properties such as the pump size, the mass flow rate, and the propellant property $R$; only the pump temperature is not included. There are three conclusions from model A:

1) It is evident from Eq. (3) that there exists a semidecaying period for the flow evolution:

$$
\tau_{d}=4 \Omega /\left[\alpha\left(S_{1} V_{1}+S_{2} V_{2}\right)\right]
$$

or

$$
\tau_{d 1}=4 \Omega /\left(\alpha S_{1} V_{1}\right), \quad \tau_{d 2}=4 \Omega /\left(\alpha S_{2} V_{2}\right)
$$

Obviously, the density evolution involves multiple time-scales, especially if different, detailed expressions for $V_{1}$ and $V_{2}$ are considered.

2) No matter how efficiently the pumps work, there is a certain amount of finite backpressure between the plates. This backpressure is represented by the second term of Eq. (3). The expression also 
indicates that with fixed pump parameters, at the final steady state, the background pressure is proportional to the mass flow rate.

3) When $S_{1}=0$, the analytical results for both short and long chambers degenerate consistently back to the results obtained in our previous work [5,7].

The pressure can be calculated from Eq. (4) with known facility properties, including cryogenic pump sticking coefficient, pump sizes, proper estimations of $V_{1}$ and $V_{2}$, and a given mass flow rate. This model illustrates that the steady background pressure is inversely proportional to the sticking coefficient when the mass flow rate is fixed. At small values of $\alpha$, a $1 \%$ difference in the coefficient may result in a significant backpressure variation, whereas for large values of $\alpha$ the normalized pressure is not very sensitive to this parameter.

\section{Model 2: Steady Detailed Kinetic Model}

This model is a steady, detailed kinetic model illustrated by Fig. 1 as well. This model considers the detailed flux balance in the flowfield, hence it can predict more accurate results than the first model. When the gas flow between the two plates reaches a final steady state, fluxes along the two directions must be exactly balanced everywhere in the chamber. This property requires several number density relations. Analysis of these flux and number density relations is the key to the formulation of this steady-flow model. One important relation for this study is the number density for a group of particles reflected from a plate with a different temperature. To preserve the equal mass flux, the following relation must hold:

$$
n_{1} \sqrt{T_{1}}=n_{2} \sqrt{T_{2}}
$$

where the subscripts 1,2 represent the incoming and reflected groups of particles, respectively [9].

With the general short chamber assumption, there are four number density relations in the flowfield, and they represent different groups of particles traveling along two directions with thermal speed characterized by the wall temperature and the pump temperature, as illustrated in Fig. 1, and they form a complete velocity distribution for the free-molecular flow between the two plates:

$$
\begin{aligned}
& f(C) \\
& = \begin{cases}n_{1+} \sqrt{\frac{m}{2 \pi k T_{w}}} \exp \left(-\frac{m C^{2}}{2 k T_{w}}\right)+n_{2+} \sqrt{\frac{m}{2 \pi k T_{p}}} \exp \left(-\frac{m C^{2}}{2 k T_{p}}\right), & C>0 \\
n_{1-} \sqrt{\frac{m}{2 \pi k T_{w}}} \exp \left(-\frac{m C^{2}}{2 k T_{w}}\right)+n_{2-} \sqrt{\frac{m}{2 \pi k T_{p}}} \exp \left(-\frac{m C^{2}}{2 k T_{p}}\right), & C<0\end{cases}
\end{aligned}
$$

where $n_{1+}$ represents the number density of particles moving towards the bottom plate, with thermal velocities characterized by the wall temperature; $n_{2+}$ represents the number density of particles moving towards the bottom plate, with thermal velocities characterized by the pump temperature. Similar definitions are used for $n_{1-}$ and $n_{2-}$ to represent the number densities of particles moving upwards. From the preceding velocity distribution function, it is evident that the flow between the two plates cannot be described using a Maxwellian distribution, and the average velocity is generally not zero.

Following the reflection relation, Eq. (10), the velocity distribution function, Eq. (11) and F ig. 1, there are four number density relations existing in the flowfield as a result of reflections on both plates, and a set of solutions is obtained from these relations.

$$
\begin{gathered}
n_{1+}=n_{\mathrm{in}}+n_{1-}\left(1-S_{1} / S_{c}\right)+n_{2-}\left(1-S_{1} / S_{c}\right) \sqrt{T_{p} / T_{w}} \\
n_{2+}=n_{1-} S_{1} / S_{c}(1-\alpha) \sqrt{T_{w} / T_{p}}+n_{2-}(1-\alpha) S_{1} / S_{p}
\end{gathered}
$$

$$
\begin{gathered}
n_{1-}=n_{1+}\left(1-S_{2} / S_{c}\right)+n_{2+}\left(1-S_{2} / S_{c}\right) \sqrt{T_{p} / T_{w}} \\
n_{2-}=n_{1+} S_{2} / S_{c}(1-\alpha) \sqrt{T_{w} / T_{p}}+n_{2+}(1-\alpha) S_{2} / S_{p}
\end{gathered}
$$

where $n_{\text {in }}=\dot{m} /\left(m S_{c} \sqrt{2 R T_{w} / \pi}\right)$, and it is the number density of inflow gas through the top end. Further denote $M=$ $\left(1-S_{1} / S_{c}\right)\left(1-\alpha S_{2} / S_{c}\right), \quad N=(1-\alpha) S_{1} / S_{c}\left(1-\alpha S_{2} / S_{c}\right), \quad$ and after straightforward derivations, the final solutions for the steady free-molecular flow between the two plates are

$$
\begin{gathered}
n_{1+}=n_{\text {in }}(1-N) /(1-N-M) \\
n_{2+}=n_{\text {in }} N \sqrt{T_{w} / T_{p}} /(1-N-M) \\
n_{1-}=n_{\text {in }}\left(1-S_{2} / S_{c}\right) /(1-N-M) \\
n_{2-}=n_{\text {in }} S_{2} / S_{c}(1-\alpha) \sqrt{T_{w} / T_{p}} /(1-N-M)
\end{gathered}
$$

The ratios among these number densities are $n_{1+}: n_{2+}: n_{1-}: n_{2_{-}}=$ $1-N: N \sqrt{T_{w} / T_{p}}: 1-S_{2} / S_{c}: S_{2} / S_{c}(1-\alpha) \sqrt{T_{w} / T_{p}}$, and any of these four groups of particles can be the dominating group. The normalized background mean velocity and the pressure corresponding to experimental measurements are as follows:

$$
\frac{U}{\sqrt{2 R T_{w}}}=\frac{\alpha\left(S_{2} / S_{c}\right)}{\sqrt{\pi}\left\{2-N-S_{2} / S_{c}+\left[N+(1-\alpha) S_{2} / S_{c}\right] \sqrt{T_{w} / T_{P}}\right\}}
$$

$$
\begin{aligned}
& \frac{P_{b} S_{c}}{\dot{m} \sqrt{2 R T_{w}}} \\
& =\frac{\sqrt{\pi}\left\{2-N-S_{2} / S_{c}+\left[N+(1-\alpha) S_{2} / S_{c}\right] \sqrt{T_{w} / T_{P}}\right\}}{4(1-N-M)}
\end{aligned}
$$

Note the backpressures are calculated with $P_{b}=n k T_{w}$ because an ionization gauge is generally used in experiments to measure the background pressure.

The preceding results are expressions for general short chamber situations. By simply setting $T_{p} / T_{w}=1$, the results for long wall situations are obtained:

$$
\begin{gathered}
n_{1+}=n_{\text {in }}(1-N) /(1-N-M) \\
n_{2+}=n_{\text {in }} N /(1-N-M) \\
n_{1-}=n_{\text {in }}\left(1-S_{2} / S_{c}\right) /(1-N-M) \\
n_{2-}=n_{\text {in }} S_{2} / S_{c}(1-\alpha) /(1-N-M)
\end{gathered}
$$

We want to point out that the incoming flux is implicitly included in Eq. (12), whereas the outflux relation is implicitly satisfied by the four number relations. Hence, even though we do not explicitly list the flux balance equation, the flux into the chamber via the top plate and out of the chamber via two pumps is implicitly balanced. The validity of this model will be illustrated via comparisons with numerical simulations.

It is interesting and necessary to verify the compatibility of these new results with our previous results of free-molecular flows 
between two plates equipped with one-side pumps on the bottom plate only [ $\underline{5}$,7]. When $S_{1}=0, M=1-\alpha S_{2} / S_{c}, N=0, n_{2+}=0$, $n_{1+}=n_{\text {in }} /\left(\alpha S_{2} / S_{c}\right), n_{1-}=\left(1-S_{2} / S_{c}\right) n_{\text {in }} /(\alpha s)$, and $n_{2-}=[(1-\alpha) / \alpha] \sqrt{T_{w} / T_{p}} n_{\text {in }}$. Hence, the new analytical results degenerate consistently to our previous results as expected and a perfect compatibility is obtained.

\section{Model 3: Steady Detailed Kinetic Model with Wall Effect}

This model is an advanced steady kinetic model illustrated by Fig. 2. Different from model 2, this model considers the sidewall effect using a geometry parameter $\chi$. With a side wall, the incoming number density at one chamber end can be attributed to two effects: a fraction of particles travels from side wall to the chamber end with a transportation percentage of $\epsilon$, and a fraction of particles travels from the other chamber end without any collisions with the chamber wall with a transport percentage of $\chi[5,6]$. It is not necessary to evaluate the first parameter $\epsilon$ because it will be canceled, whereas the evaluation of the other key parameter $\chi$ involves two steps: first, compute the solid angle at one specific point on one chamber end subtended by the other chamber end, with the following solid angle formula [1]ㄹ:

$$
\Omega=\int_{s} \frac{\boldsymbol{r} \cdot \boldsymbol{n} \mathrm{d} S}{r^{3}}=\int_{0}^{2 \pi} H \mathrm{~d} \phi \int_{0}^{R} \frac{\eta^{2} \mathrm{~d} \eta}{\left(r^{2}+H^{2}-2 r \eta \cos \eta+\eta^{2}\right)^{3 / 2}}
$$

The final format of this integral involves the Heuman's Lambda function and the complete elliptic integral of the first kind [10]. The second step is to average the solid angle over all points on this chamber end. Though there is an analytical result for the first step, it is extremely difficult to obtain an analytical expression for the second step, which is required to evaluate the analytical results from this model. Hence, the transportation coefficient $\chi$ is evaluated numerically. For the two numerical simulation cases in this study, $\chi$ equals 0.05 and 0.63 for cylindrical chamber $L / R=9 / 3$ and $0.9 / 3$, respectively $[5,6]$.

In this model, we assume at chamber ends A and B, there are two groups of incoming particles and two groups of reflected particles, as illustrated in Fig. 2. Hence, the velocity distribution functions can be expressed as follows:

$$
\begin{aligned}
& f_{A}(C)= \begin{cases}n_{A 1-} \sqrt{\frac{m}{2 \pi k T_{w}}} \exp \left(-\frac{m C^{2}}{2 k T_{w}}\right)+n_{A 2-} \sqrt{\frac{m}{2 \pi k T_{p}}} \exp \left(-\frac{m C^{2}}{2 k T_{p}}\right), & C>0 \\
n_{A 1+} \sqrt{\frac{m}{2 \pi k T_{w}}} \exp \left(-\frac{m C^{2}}{2 k T_{w}}\right)+n_{A 2+\sqrt{\frac{m}{2 \pi k T_{p}}} \exp \left(-\frac{m C^{2}}{2 k T_{p}}\right),} \quad C<0\end{cases}
\end{aligned}
$$

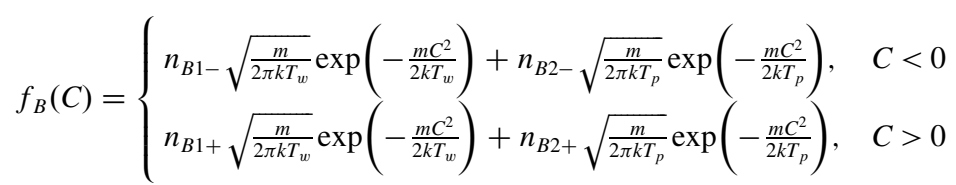

At both chamber ends, the incoming number densities for particles with a thermal speed characterized by the wall temperature consist of two parts; one from the other chamber end and one from the sidewall:

$$
n_{A 1+}=\chi n_{B 1-}+\epsilon n_{w}, \quad n_{B 1+}=\chi n_{A 1-}+\epsilon n_{w}
$$

The number densities of particles with a thermal speed characterized by the pump temperature are

$$
n_{A 2+}=\chi n_{B 2-}, \quad n_{B 2+}=\chi n_{A 2-}
$$

There are four reflection relations at both chamber ends:

$$
\begin{gathered}
n_{A 1-}=\left(1-S_{1} / S_{c}\right) n_{A 1+}+n_{A 2+}\left(1-S_{1} / S_{c}\right) \sqrt{T_{p} / T_{w}}+n_{\mathrm{in}} \\
n_{A 2-}=S_{1} / S_{c}(1-\alpha) \sqrt{T_{w} / T_{p}} n_{A 1+}+n_{A 2+} S_{1} / S_{c}(1-\alpha) \\
n_{B 1-}=\left(1-S_{2} / S_{c}\right) n_{B 1+}+n_{B 2+}\left(1-S_{2} / S_{c}\right) \sqrt{T_{p} / T_{w}} \\
n_{B 2-}=S_{2} / S_{c}(1-\alpha) n_{B 1+} \sqrt{T_{w} / T_{p}}+n_{B 2+} S_{2} / S_{c}(1-\alpha)
\end{gathered}
$$

Finally, the mass flux into the chamber is balanced by the mass flux absorbed by the two pumps:

$$
\dot{m}=n_{A 1+} \alpha S_{1} m V_{w}+n_{A 2+} \alpha S_{1} m V_{p}+n_{B 1+} \alpha S_{2} m V_{w}+n_{B 2+} \alpha S_{2} m V_{p}
$$

The final solutions for the above nine equations are quite complex. Denote $M=1-S_{1} / S_{c}, N=\left(1-S_{1} / S_{c}\right) \sqrt{T_{p} / T_{w}} \chi, P=S_{1} /$ $S_{c}(1-\alpha) \sqrt{T_{w} / T_{p}}, \quad Q=\chi S_{1} / S_{c}(1-\alpha), \quad R=1-S_{2} / S_{c}, \quad S=\left(1-S_{2} / S_{c}\right) \chi \sqrt{T_{p} / T_{w}}, \quad K=S_{2} / S_{c}(1-\alpha) \sqrt{T_{w} / T_{p}}, \quad T=\chi S_{2} /$ $S_{c}(1-\alpha), C=\alpha S_{1} V_{w}, D=\alpha S_{1} V_{p} \chi, E=\alpha S_{2} V_{w}, F=\alpha S_{2} V_{p} \chi, \Phi=(1+\chi M-\chi S P)(1-T Q)-(\chi S Q-\chi N) T P, \Psi=(1-T Q)(1+$ $\chi R)+(\chi S Q-\chi N) K$, then the solutions can be expressed as

$$
n_{B 1+}=\frac{(\dot{m} / m)+(1-T Q)(\chi / \Phi)[C+F P+(D+F Q)(T P) /(1-T Q)] n_{\text {in }}}{(\Psi / \Phi)[C+F P+(D+F Q)(T P) /(1-T Q)]+(D+F Q) K /(1-T Q)+E}
$$




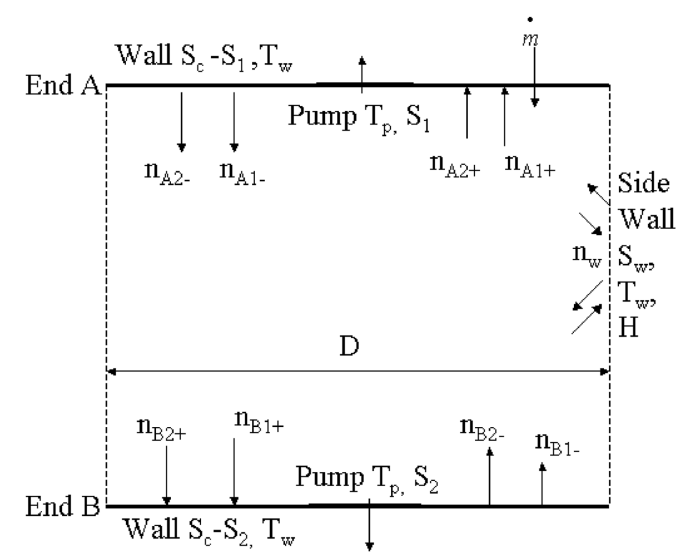

Fig. 2 Illustration of model 3 (not to scale).

$$
\begin{gathered}
n_{A 1+}=\frac{\Psi}{\Phi} n_{b 1+}-\frac{\chi}{\Phi} n_{\text {in }}(1-T Q) \\
n_{B 2-}=\frac{K}{1-T Q} n_{B 1+}+\frac{T P}{1-T Q} n_{A 1+} \\
n_{A 2-}=P n_{A 1+}+Q n_{B 2-}
\end{gathered}
$$

$n_{A 1-}=M n_{A 1+}+N n_{B 2-}+n_{\text {in }}, \quad n_{B 1-}=R n_{B 1+}+S n_{A 2-}$

$$
n_{A 2+}=\chi n_{B 2-}, \quad n_{B 2+}=\chi n_{A 2-}
$$

The normalized average background velocity and pressure are evaluated by averaging the values at two chamber ends:

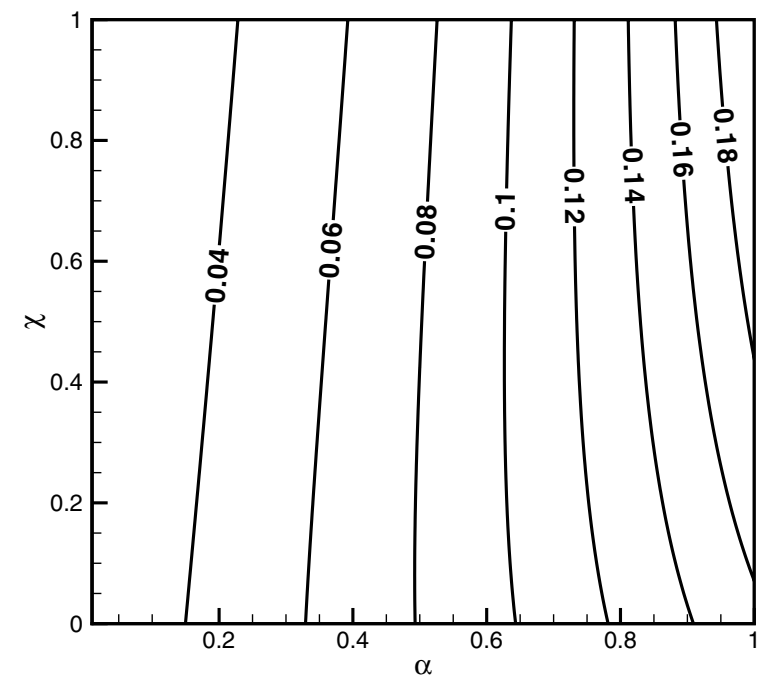

Fig. 4 Contours of velocity distribution $U_{\mathrm{av}} / \sqrt{2 R T_{w}}\left(S_{1} / S_{c}=0.2\right.$, $\left.S_{2} / S_{c}=0.4\right)$.

$$
\begin{aligned}
& \frac{P_{b} S_{c}}{\dot{m} \sqrt{2 R T_{w}}}=\frac{\sqrt{\pi}}{8 n_{\text {in }}}\left(n_{A 1+}+n_{A 2+}+n_{A 1-}+n_{A 2-}+n_{B 1+}\right. \\
& \left.\quad+n_{B 2+}+n_{B 1-}+n_{B 2-}\right)
\end{aligned}
$$

At the end of this section, we want to point out that model 3 is a relatively advanced model because it is capable of analytically estimating the chamber sidewall effects. Figures $\underline{3}$ and $\underline{4}$ show contours of average background pressure and average velocity in the chamber with different sidewall length represented by $\chi$ and absorption coefficient represented by $\alpha$. The pump sizes are fixed at $S_{1} / S_{c}=0.2$ and $S_{2} / S_{c}=0.4$. We can conclude from these two pictures that when $\alpha<0.5$, the value of $\partial(P) / \partial \chi$, which is the

$\frac{U_{\mathrm{av}}}{\sqrt{2 R T_{w}}}=\frac{n_{A 1-}+n_{B 1+}-n_{A 1+}-n_{B 1-}+\left(n_{A 2-}+n_{B 2-}-n_{A 2+}-n_{B 2-}\right) \sqrt{T_{p} / T_{w}}}{\sqrt{\pi}\left(n_{A 1+}+n_{A 2+}+n_{A 1-}+n_{A 2-}+n_{B 1+}+n_{B 2+}+n_{B 1-}+n_{B 2-}\right)}$

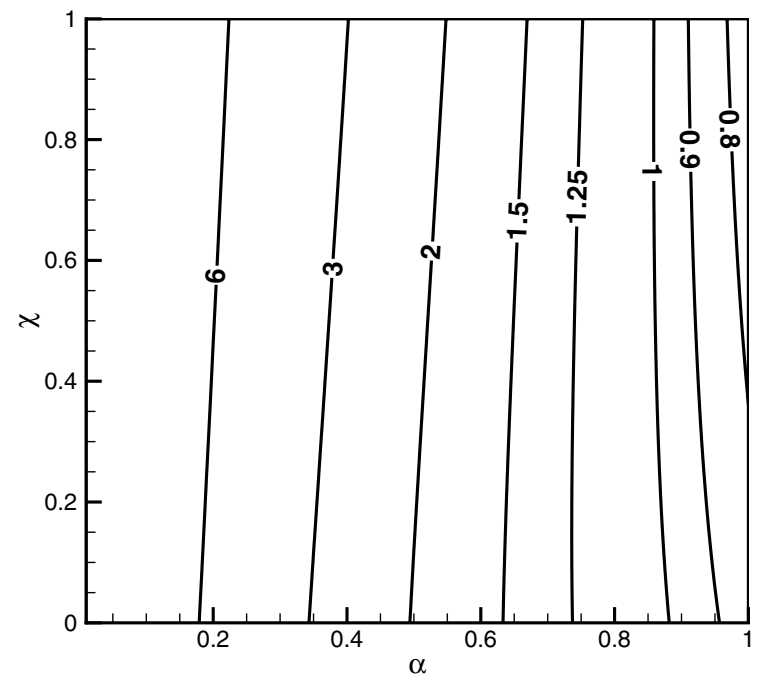

Fig. 3 Contours of pressure distribution $P_{b} S_{c} / \dot{m} \sqrt{2 R T_{w}}\left(S_{1} / S_{c}=0.2\right.$, $\left.S_{2} / S_{c}=0.4\right)$. indicator of wall length effect on the background pressure, is greater than zero; when $\alpha>0.9, \partial(P) / \partial \chi<0$; whereas in the range of 0.5 and 0.9 for $\alpha, \partial(P) / \partial \chi$ can take positive or negative values. This fact indicates that the chamber wall has a complex influence on the final flow state. This fact will be illustrated later with numerical simulations.

\section{Numerical Simulations}

For vacuum chambers equipped with distributed pumps, there are no experimental measurements available, hence the validity of these models relies on comparisons with numerical simulations. Because the flow between the two plates is free-molecular, it is ideal to validate the preceding analytical results with the direct simulation Monte Carlo (DSMC) method [11]. This study uses a specific DSMC package named MONACO [12] to perform simulations.

DSMC simulations are physically accurate by including collisions, even though collisions occur very infrequently. The DSMC method simulates collision effects in rarefied gas flow by collecting groups of particles into cells. Pairs of particles inside a cell are selected at random and collision probability is evaluated that is 
proportional to the product of the relative velocity and the collision cross section for each pair. The probability is compared with a random number to determine if that collision occurs. If so, some form of collision dynamics is performed to alter the properties of the colliding particles. The no time counter method [11] is adopted to determine the collision rate. In this study, momentum collisions, or elastic collisions, involving exchange of momentum between the participating particles are included in the DSMC method. The variable hard sphere [11] model is employed and the collision cross section of xenon is:

$$
\sigma_{e l}(X e, X e)=2.12 \times 10^{-18} \mathrm{~g}^{-2 \omega} \mathrm{m}^{2}
$$

where $\omega$ is the relative velocity and $\omega=0.12$ is related to the viscosity temperature exponent for xenon. In all elastic interactions, the collision dynamics is modeled using isotropic scattering together with conservation of linear momentum and energy to determine the postcollision velocities of the colliding particles. In total, over 140 axisymmetric simulations of background xenon gas flows in a cylindrical chamber are performed. In half of these simulations, the intermolecular collision module in the package is deactivated, whereas for the rest of simulations, the intermolecular collision module is activated. Hence, these simulation results provide both real physical flow results and ideal free-molecular flow results.

In this study, a cylindrical simulation domain is adopted and partially illustrated by Fig. 5. The top and bottom sides are the chamber ends, the left side is the symmetric axis, and the right side is the outer surface. The cylindrical end section radii are set to $3 \mathrm{~m}$, and the gap between the two plates is set to either $9 \mathrm{~m}$ or $0.9 \mathrm{~m}$, representing long and short chamber situations, respectively. To simplify the situation, we fix the top pump size by setting $S_{1} / S_{c}=0.2$. The mass flow rate into the chamber through the top plate is set to $\dot{m}=6.17 \times 10^{-7} \mathrm{~kg} / \mathrm{s}$. The pump temperature is set to $15 \mathrm{~K}$, because cyrogenic pumps are usually maintained at low temperature of $10-20 \mathrm{~K}$ in operation. The wall temperature is set to room temperature $300 \mathrm{~K}$. At the initial time step, an ambient density or vacuum condition is assumed. A mesh size of $15 \times 30$ cells is adopted in the simulation to achieve relatively high resolution. The "no concrete pumps" treatment [5] is adopted on both plates. In this approach, no specific locations on the two plates are specified as pumps. When a particle hits a plate, a random number is selected to compare with the particular area ratio $S_{1} / S_{c}$ or $S_{2} / S_{c}$. If the random number is smaller than the area ratio, then this particle hits the pump, otherwise it hits the chamber end. By following this approach, it is not necessary to generate a new mesh for each new case, and it is an effective way to simulate small pump areas. Once a particle hits a pump, then another random number is generated, if this number is smaller than the pump sticking coefficient then this particle sticks to

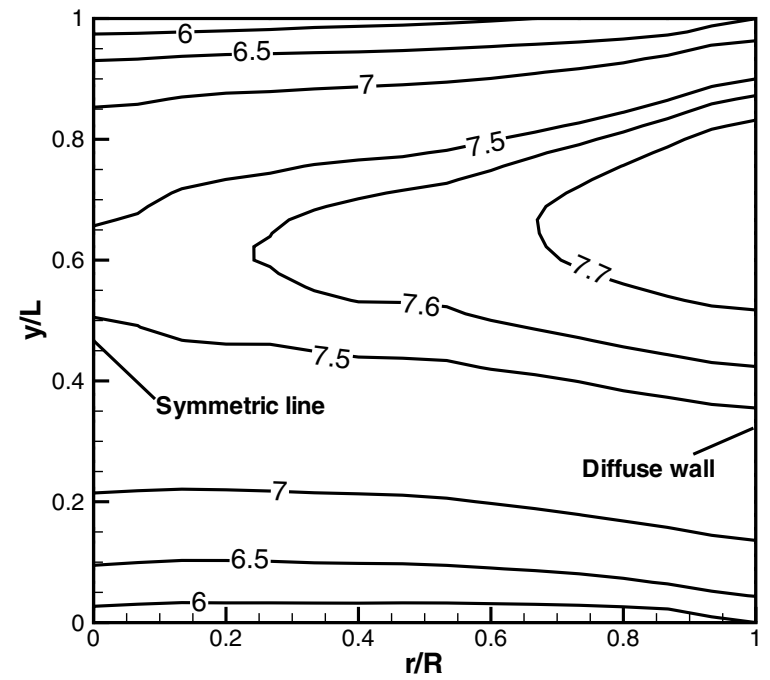

Fig. 5 Contours of mean free path, $m$, with collisions $(\alpha=0.3$, $\left.S_{2} / S_{c}=0.3, L / R=9 / 3, T_{w}=300 \mathrm{~K}, T_{p}=15 \mathrm{~K}\right)$.

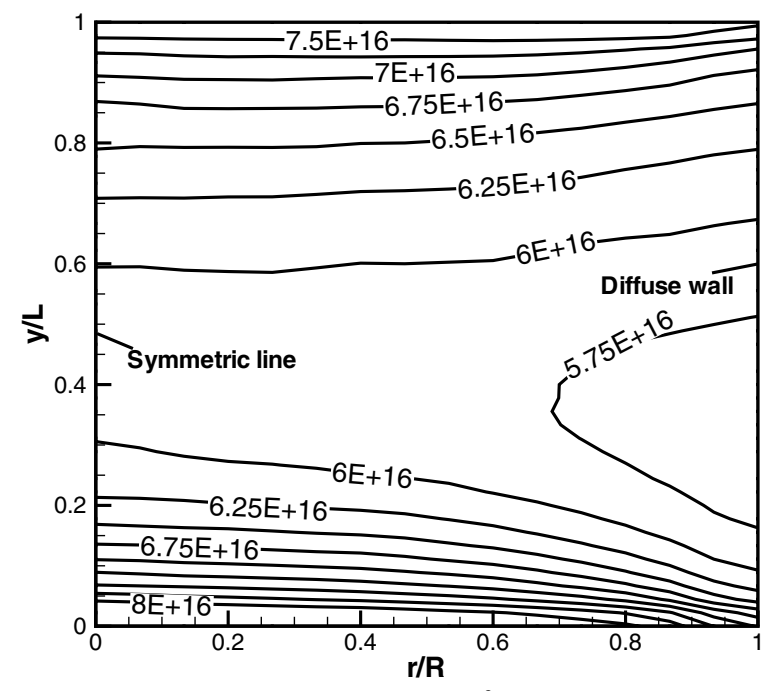

Fig. 6 Contours of number density, $\mathrm{m}^{-3}$, with collisions $(\alpha=0.4$, $\left.S_{2} / S_{c}=0.6, L / R=9 / 3, T_{w}=300 \mathrm{~K}, T_{p}=15 \mathrm{~K}\right)$.

the pump and is removed from the simulation, otherwise the particle is reflected from the pump with a thermal velocity characterized by the low pump temperature. In the DSMC simulations, particles are introduced into the simulation domain through one chamber end with given mass flow rate and given thermal speeds characterized by the wall temperature. The time step and particle weight, which represent the amount of real molecules with one particle, vary in different cases. Most simulation details are the same as the simulations in our previous study $[5,7]$ except there is an extra small pump on the top plate.

\section{A. Evaluation of Average Quantities}

Walker's experimental results [1] show that the background pressure depends on pump locations, but to include the pump location effect will need a two-dimensional model, and it is not the goal of this study. The primary concern of this study is to provide formulas to estimate the average background pressure and average background velocity in the chamber. Hence, the detailed background flowfield structures are neglected. At each time step, an average density and an average velocity for the whole domain are computed by counting all particles in the simulation domain and averaging their velocities:

$$
\begin{gathered}
\rho(t)=\left(\sum\left(w_{i} N_{i}\right)\right) m / \Omega \\
U(t)=\left(\sum U_{j}\right) / N
\end{gathered}
$$

where $N_{i}$ is the total number of particles used in $i$ th cell in the DSMC simulation; $U_{j}$ and $N$ are the $j$ th particle velocity and total number of particles in the domain.

\section{B. Comment on Accuracy}

The major purpose of this study is to provide several analytical expressions to evaluate the average background pressure and average background flow velocities. Because the flow is freemolecular, the DSMC is an accurate simulation method to provide accurate flow solutions. By counting all particles in the chamber and averaging their velocities, the preceding two equations provide accurate average results in the whole chamber. By including collisions, these DSMC simulations are expected to provide more accurate results than the analytical results. By neglecting collisions, other DSMC simulations are expected to provide exact freemolecular flow results at extreme conditions which are closer to analytical results. Hence, these particle simulation results will be 


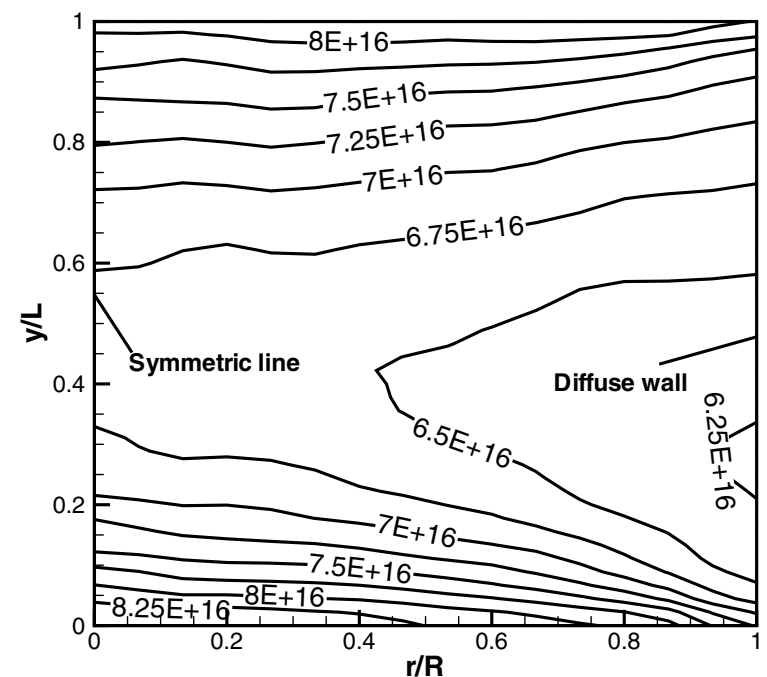

Fig. 7 Contours of number density, $\mathrm{m}^{-3}$, without collisions $(\alpha=0.4$, $\left.S_{2} / S_{c}=0.6, L / R=9 / 3, T_{w}=300 \mathrm{~K}, T_{p}=15 \mathrm{~K}\right)$.

used to compare the effectiveness of the analytical models. The detailed distributions in these two-dimensional simulations may be affected by subtle changes in boundary treatments, but the average number density and average velocity in the chamber are not sensitive to these changes in boundary treatment. This scheme was successfully applied in our previous studies [5,7].

Figure 5 shows the contours of mean free path for the case $\alpha=0.4, \overline{S_{2}} / S_{c}=0.3$ and with particle collisions. It is obvious that in the flowfield, the mean free path is larger than $6 \mathrm{~m}$, which is the diameter of the cylindrical simulation domain. Hence, a freemolecular flow will be achieved. Most of the simulations in this study have a higher degree of rarefaction than this case by a larger value of $\alpha$ or $S_{2} / S_{c}$.

Figures $\underline{6}$ and $\underline{7}$ show two number density distributions from DSMC simulations for a long chamber. Gas flows into the chamber from the top plate, and with a consideration of reflections, both the top and the bottom plates are essentially sources where particles effuse into the chamber. Though this study is not focused on flowfield details, these two typical figures are helpful to understand the results. The simulation parameters for these two figures are the same except that the intermolecular collision module is turned off for Fig. 7. Hence, Fig. 7 is a free-molecular flow result. It is obvious that these two figures are quite similar, but the first figure has a lower average density. The similarity is a result of the same chamber geometry, whereas the difference arises by including or not including

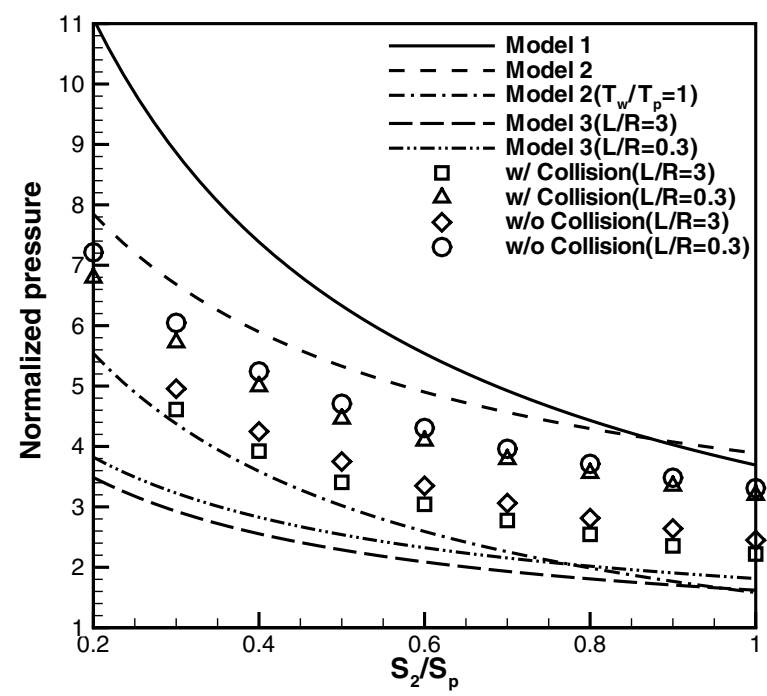

Fig. 9 Average density (normalized by $\left.\left(\dot{m} / m S_{c}\right) \sqrt{2 \pi / R T_{w}}, \alpha=0.4\right)$.

molecular collisions. By including collisions, particles are impeded in crossing the dense layers close to both chamber ends, they are reflected back, and absorbed by the pumps on the chamber ends. Whereas for free-molecular flow, in the second figure, particles are free to pass through the dense layers close to both ends and enter the middle of the chamber. Hence, for free-molecular flows, with less absorption effects, a higher average number density is anticipated under some specific parameter combinations, and these results will be further illustrated later.

Figure $\underline{8}$ shows several average density evolution results from model 1 and DSMC simulations. From this figure, it is evident that both the analytical results and the DSMC simulation results predict several semidecaying periods and final steady densities. The freemolecular DSMC simulation yields a higher final average number density than that with molecular collisions. With collisions, the semidecaying period is shorter because collisions aid the process of forming an equilibrium state. The analytical results overpredict the semidecaying period, and the difference can be attributed to the simplified velocity $V_{1}=V_{2}=V_{w}$. In general, model 1 is the crudest model without any detailed flux balance at all.

Figures $\underline{9}$ and 10 show two series of average backpressure with varying $S_{2} / S_{c}$, and $\alpha=0.4,0.8$, respectively. Analytical results from the three models and DSMC simulations are included. It is evident that all of the models predict the correct trends, and for $\alpha=0.8$ the gaps in both analytical and numerical simulations

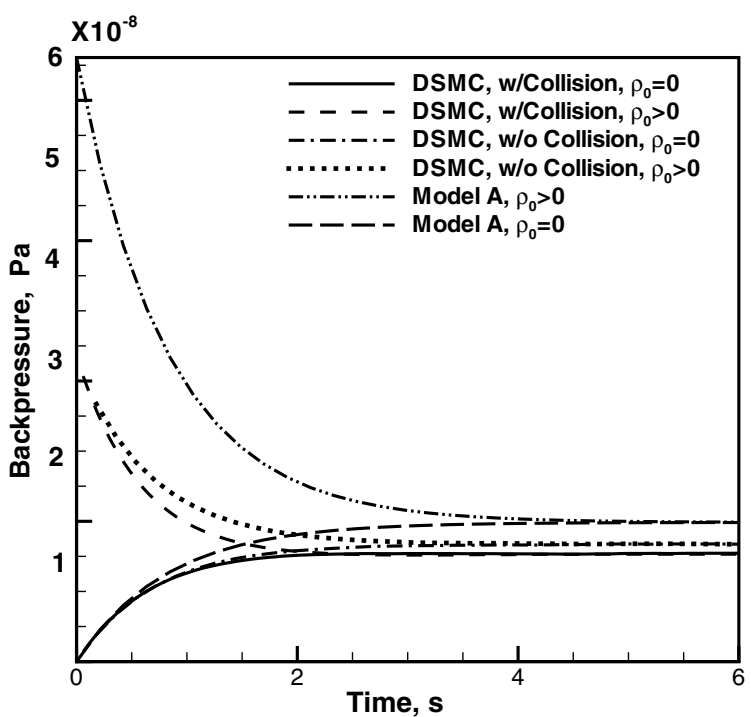

Fig. 8 Comparison of evolution history $\left(\alpha=0.4, S_{2} / S_{c}=0.5\right)$.

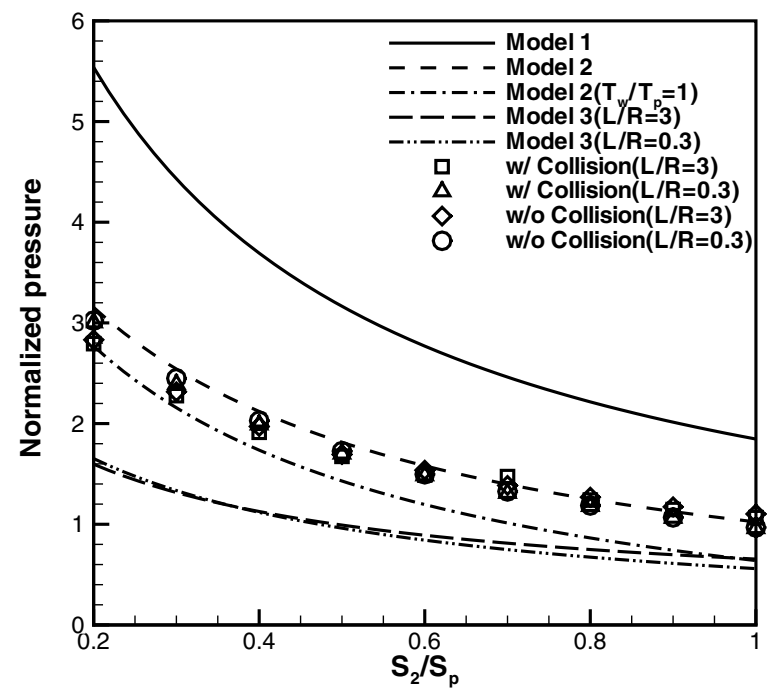

Fig. 10 Average density (normalized by $\left.\left(\dot{m} / m S_{c}\right) \sqrt{2 \pi / R T_{w}}, \alpha=0.8\right)$. 


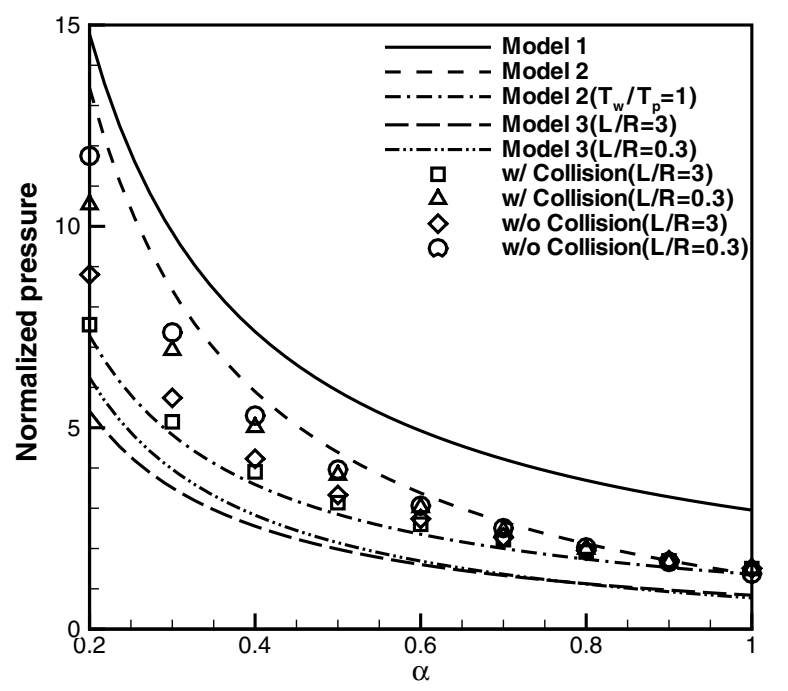

Fig. 11 Average density (normalized by $\left(\dot{m} / m S_{c}\right) \sqrt{2 \pi / R T_{w}}$, $\left.S_{2} / S_{c}=0.4\right)$.

decrease. Figure 9 indicates that the free-molecular DSMC simulations yield higher average densities than those simulations without molecular collisions, whereas in Fig. 10, all simulation results are quite close. Models 1 and 3 have improved performance when $S_{2} / S_{p}$ increases. Another important difference between these two figures is that in the first figure with $\alpha=0.4<0.5$, no intersection occurs in both the simulation data and model 3 results; whereas in the second picture with $\alpha=0.8$, the analytical long chamber wall and short chamber wall curves intersect, and the simulation data intersect as well. These results are consistent with our previous observation about $\partial() / \partial(\chi)$ from Fig. 3. Hence, though model 3 underpredicts the average background pressure, it is capable of quantifying the effects of chamber wall length.

Figures 11 and 12 show two series of average number density

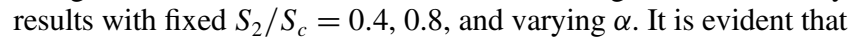
all of the models predict the correct trends and for $S_{2} / S_{c}=0.8$, both analytical and number simulation results are in good agreement. In general, model 2 still has the best performance. It is interesting to note that model 3 predicts that at $\alpha=0.6-0.9$ the short chamber curve and the long chamber curve intersect, and numerical simulations confirm these intersections, more obviously shown in Fig. 12. These $\alpha$ values at the intersections are different from the results obtained in our previous study of single pump situations due to the secondary small

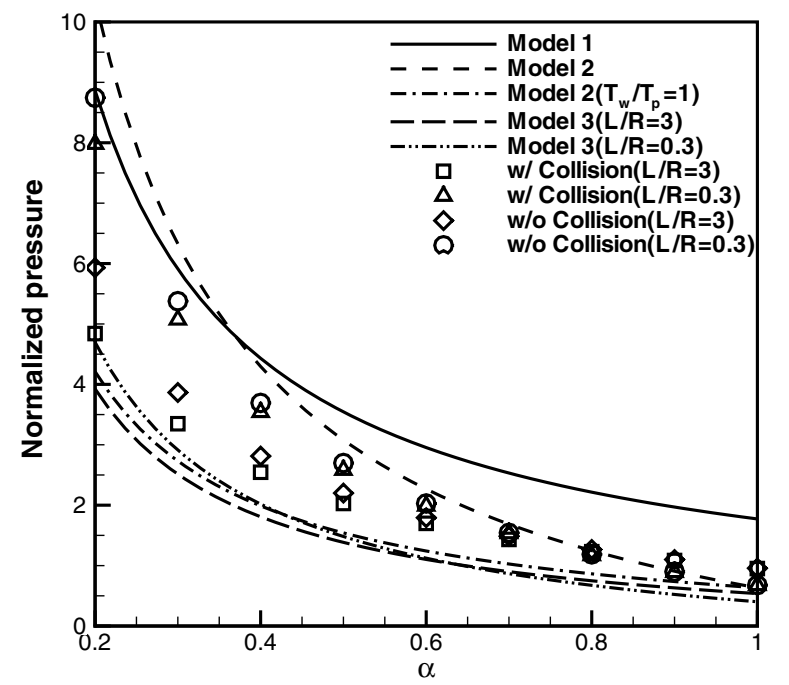

Fig. 12 Average density (normalized by $\left(\dot{m} / m S_{c}\right) \sqrt{2 \pi / R T_{w}}$, $\left.S_{2} / S_{c}=0.8\right)$.

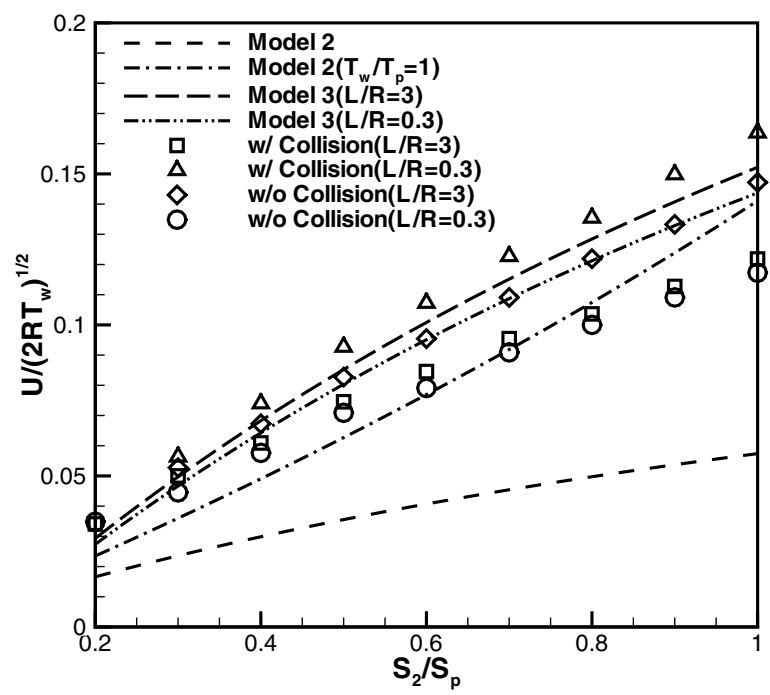

Fig. 13 Average velocity ratio $U / \sqrt{2 R T_{w}}, \alpha=0.4$.

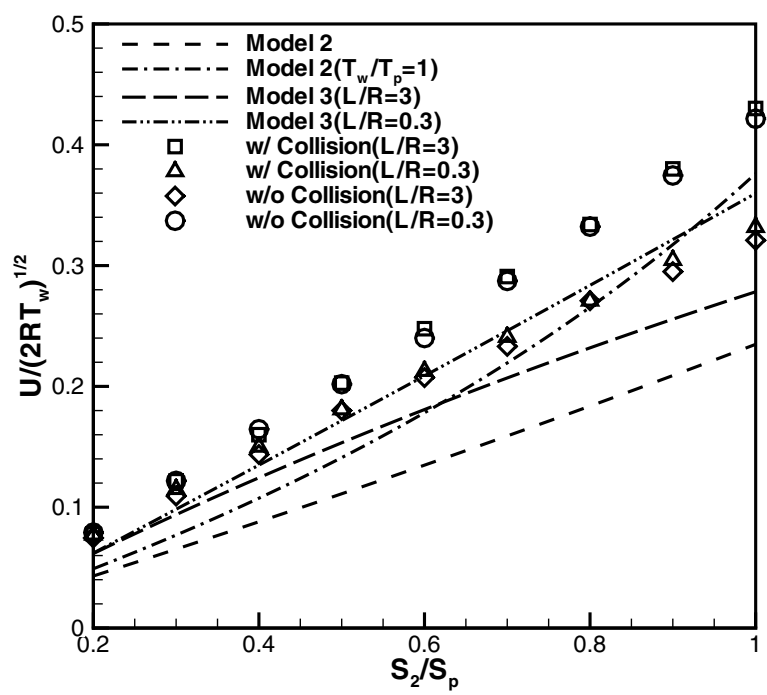

Fig. 14 Average velocity ratio $U / \sqrt{2 R T_{w}}, \alpha=0.8$.

pump. It is difficult to determine this value analytically with the complex results from model 3 .

Figures 13 and 14 show two series of average background velocity results with fixed $\bar{\alpha}=0.4,0.8$, but varying $S_{2} / S_{c}$. It is evident that all of the models predict the same trends as the numerical simulations. For $\alpha=0.8$, the free-molecular simulations have lower average velocity values than those simulations with molecular collisions. The reason for this result is, by neglecting the collisions, more particles are reflected back from the bottom pump with negative velocities. Hence, a lower average background velocity is expected. For these velocity results, model 2 with $T_{p} / T_{w}=1$ and model 3 have better performance than model 2 when compared with numerical simulation results.

Figures 15 and 16 show two series of average background velocity results with fixed $\bar{S}_{2} / S_{c}=0.4,0.8$, and varying $\alpha$. All analytical models predict the correct trends. For most combinations of parameters, the simulations without considering collisions have a lower average background velocity than those simulations with considering molecular collisions. The reason is simple: because the large pump is located at the far chamber end, without considering collisions, all particles reflected at the pump will move to the other end or wall with negative velocities. With considering collisions, a portion of those reflected particles will move back to the primary pump and be absorbed again, due to the dense collisions around the primary pump, hence, less negative velocities will be included in the computation for the average velocity. 


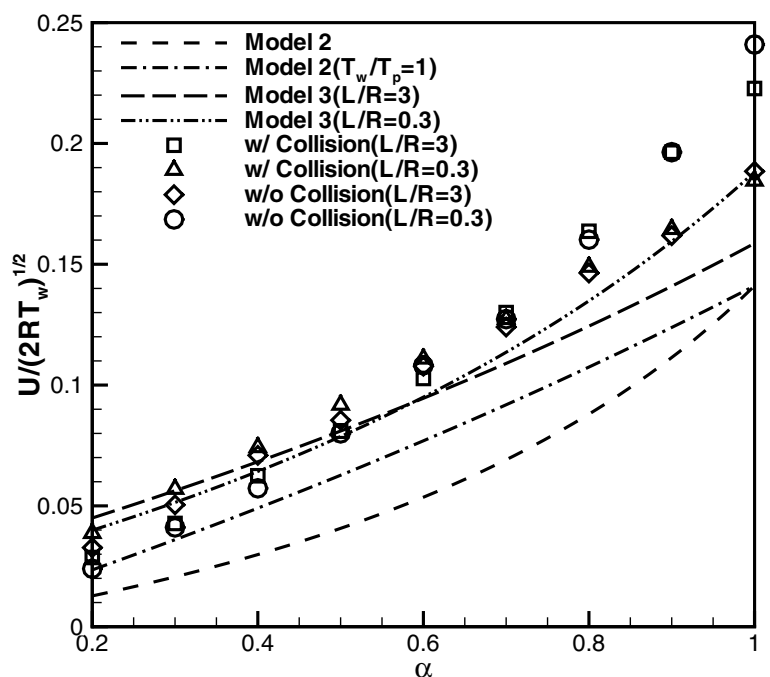

Fig. 15 Average velocity ratio $U / \sqrt{2 R T_{w}}, S_{2} / S_{c}=0.4$.

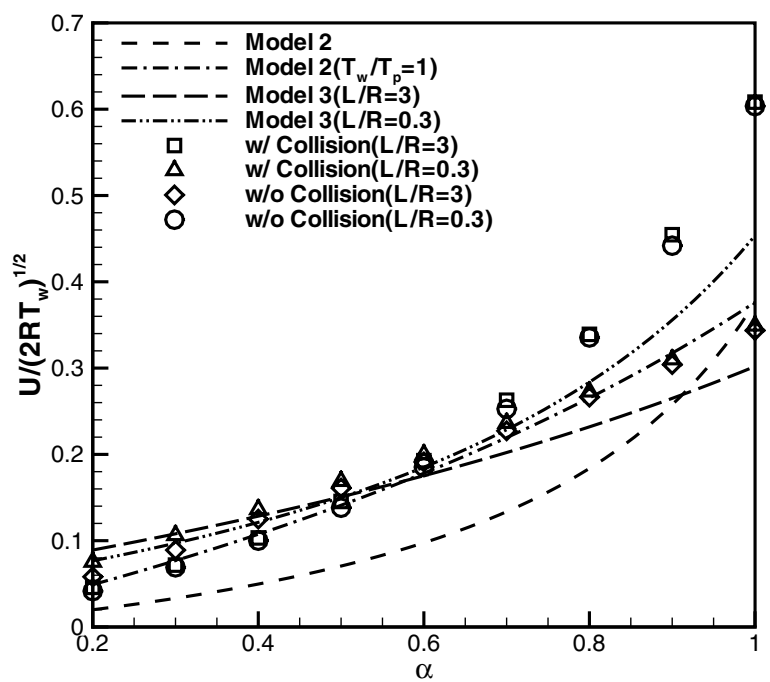

Fig. 16 Average velocity ratio $U / \sqrt{2 R T_{w}}, S_{2} / S_{c}=0.8$.

\section{Conclusions}

In this study, we proposed three models to study the average background pressure and the average velocity between two plates both equipped with cyrogenic pumps. The results indicate that the analytical results are more complicated than the situations in our previous study. However, the results from this study are more general and can degenerate consistently to our previous study.

To validate the analytical results from the three models, we performed about 140 DSMC simulations, with and without intermolecular collisions. Generally, the analytical and numerical results have the same trends, whereas models 2 and 3 offer quite good agreement to the DSMC simulations.

Similar to our previous study, several conclusions can be drawn for the background free-molecular flows: the background flow is not static with zero average velocity and cannot be described as a full Maxwellian distribution, and the long semidecaying period prohibits a full 3-D simulation of plume flows in the whole chamber. Hence, extra attention is necessary for the background flow construction when simulating plume flows in large vacuum chambers with a particle approach such as the DSMC method.

\section{Acknowledgments}

The authors gratefully acknowledge funding for this work from the U.S. Air Force Office of Scientific Research under grant FA955005-1-0042. This work was carried out and partially completed at the University of Michigan.

\section{References}

[1] Walker, M. L., "Effects of Facility Backpressure on the Performance and Plume of a Hall Thruster, Ph.D. Thesis," Ph.D. Dissertation, Aerospace Engineering Dept., Univ. of Michigan, Ann Arbor, MI, 2005.

[2] Hargus, W. A., and Reed, G., "Air Force Clustered Hall Thruster Program," AIAA Paper 2002-3678, July 2002.

[3] Beal, B. E., "Clustering of Hall Effect Thrusters for High-Power Electric Propulsion," Ph.D. Dissertation, Aerospace Engineering Dept., Univ. of Michigan, Ann Arbor, MI, 2004.

[4] Biagioni, L., Boccaletto, L., Marcuccio, L., Nicolini, S., Servi, D., and Andrenucci, M., "Large Space Simulator for Electric Propulsion Testing: Design Requirements and Engineering Analysis," AIAA Paper 2000-3750, July 2000.

[5] Cai, C., "Theoretical and Numerical Studies of Plume Flows in Vacuum Chambers," Ph.D. Dissertation, Aerospace Engineering Dept., Univ. of Michigan, Ann Arbor, MI, 2005.

[6] Cai, C., Boyd, I. D., and Sun, Q., "Free Molecular Background Flow in a Vacuum Chamber Equipped with Two-Sided Pumps," Journal of Vacuum Science and Technology A (Vacuum, Surfaces, and Films), Vol. 24, No. 1, 2006, pp. 9-19.

[7] Cai, C., Boyd, I. D., and Sun, Q., "Rarefied Background Flow in a Vacuum Chamber Equipped with One-Sided Pumps," Journal of Thermophysics and Heat Transfer, Vol. 20, No. 3, July-Sept. 2006, pp. 524-535.

[8] Meyer, J. W., Loane, J. T., Hallock, G. A., Wiley, J. C., and Dawbarn, R. A., "Hall Thruster Plume Signal Transmission Testing in the AEDC 12V Vacuum Chamber," AIAA Paper 2005-4049, July 2005.

[9] Gombosi, T. I., Gaskinetic Theory, Cambridge Univ. Press, New York, 1994.

[10] Narasimha, R., "Orifice Flow of High Knudsen Number," Journal of Fluid Mechanics, Vol. 10, Oct. 1961, pp. 371-384.

[11] Bird, G. A., Molecular Gas Dynamics and the Direct Simulation of Gas Flows, Oxford Univ. Press, New York, 1994.

[12] Dietrich, S., and Boyd, I. D., "Scalar and Parallel Optimized Implementation of the Direct Simulation Monte Carlo Method," Journal of Computational Physics, Vol. 126, No. 2, July 1996, pp. 328342 . 